\title{
Deflection Tomography Reconstruction Based on Diagonal Total Variation
}

\author{
Huaxin $\mathrm{Li}^{\mathrm{a}}$ Jinxiao Pan \\ School of Information and Communication Engineering, North University of China, TaiYuan 030051, China
}

\begin{abstract}
In view of the shortages of the reconstruction algorithm based on Total Variation (TV) minimum under the framework of measured field compressed sensing, we study the measured field sparse representation method and solving method of optimization equation, and propose the measured field reconstruction algorithms based on Diagonal Total Variation (DTV). When there is no obvious change in the reconstruction iteration of $\mathrm{TV}$, gradient transformation of diagonal direction is introduced, the multi-directional information is used to obtain a more sparse representation of the measured field in the reconstruction. Under the condition of sparse projections, experimental results of this algorithm are demonstrated and compared with the results from the TV method. Comparisons show that this method can reconstruct high-quality measured field.
\end{abstract}

\section{Introduction}

In the field of complex flow field display and measurement, the optical deflection tomography has become a powerful and practical measurement technology due to a series of features such as strong anti-interference ability, low sensitivity and non-contact measurement. However, the method of obtaining the deflection projection of unsteady complex flow field determines that the reconstruction can only be carried out under the condition of spare projection. The method cannot satisfy the reconstruction under the condition of incomplete data, so the application of deflection tomography technology is restricted.

The reconstructed algorithm is classified into two categories: transform algorithm ${ }^{[1]}$ and series expansion algorithm ${ }^{[2]}$. The former includes Abel inverse transformation method ${ }^{[3]}$, direct inverse Radon transformation method ${ }^{[4]}$ and filtered back projection (FBP) method ${ }^{[5-6]}$. The latter mainly includes direct algebraic algorithm based on finite series expansion and algebraic reconstruction technique(ART) ${ }^{[7]}$. ART allow direct computational reconstruction from a limited data set, which may provide a restricted view, a limited number of projections, or incomplete projections. However, under extreme undersampled conditions, this kind of reconstruction algorithm cannot reconstruct the high precision measured field .

This paper combines compressed sensing (CS) theory, proposes a reconstruction algorithm based on diagonal total variation(DTV). The reconstruction algorithm based on TV which only uses $\mathrm{x}$-coordinate and y-coordinate gradient transform as its sparse representation approach hasn't made the best of the directivity of measured field. Based on this, we bring in DTV which uses the diagonal direction gradient to constraint reconstructed field and is operated when the reconstructed field has no change after a set number of TV gradient descent. Through numerical simulation we find that the proposed algorithm can reconstruct high-quality measured field, which is more suitable when the sparse sampling.

\section{Algorithm Analyses}

\subsection{Convert deflected projection information}

Figure 1 represents a 2-D phase object where the refractive index distribution is $\widehat{n}(x, y), \hat{n}(x, y)$ can be expressed as

$$
\widehat{n}(x, y)=n_{0}+f(x, y)
$$

$n_{0}$ is the refractive index of the surroundings. The $x^{\prime}-y^{\prime}$ axes specify a coordinate system rotated by an angle $\theta$, with respect to that specified by $x-y$. When the incident ray parallel to the $x^{\prime}$ axis passes through the flow field, $\varphi\left(y^{\prime}, \theta\right)$ the deflection angle of the ray along the $y^{\prime}$ direction can be expressed as ${ }^{8}$

$$
\varphi\left(y^{\prime}, \theta\right)=\frac{1}{n_{0}} \int_{-\infty}^{\infty} \frac{\partial f(x, y)}{\partial y^{\prime}} d x^{\prime}
$$

While in phase tomography, the optical path length difference $\phi\left(y^{\prime}, \theta\right)$ can be expressed as

$$
\phi\left(y^{\prime}, \theta\right)=\int_{l} f(x, y) d l
$$

and $l$ is the optical path through the measured field. When the deflection angle is small, the light can be approximately straight in the measured field.

\footnotetext{
${ }^{a}$ Corresponding author: li_huaxin@nuc.edu.cn
} 


$$
\begin{aligned}
& \frac{\partial \phi\left(y^{\prime}, \theta\right)}{\partial y^{\prime}}=\frac{\partial}{\partial y^{\prime}} \int_{l} f(x, y) d l \\
& =\int_{-\infty}^{\infty} \frac{\partial f(x, y)}{\partial y^{\prime}} d x^{\prime}=n_{0} \varphi\left(y^{\prime}, \theta\right)
\end{aligned}
$$

So, by integration operation, we can convert the deflected projection into the optical path difference projection data $^{[9]}$,

$$
\phi\left(y^{\prime}, \theta\right)=\int_{-\infty}^{\infty} n_{0} \varphi\left(y^{\prime}, \theta\right) d y^{\prime}
$$

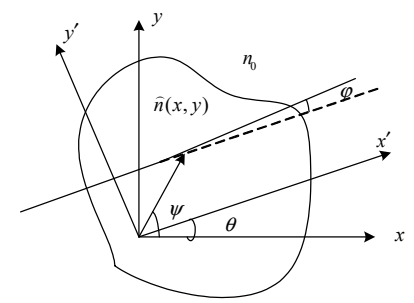

Figure 1. Schematic diagram of the beam deflection and the coordinate system; $\theta$ is the viewing angle; $\varphi$ is the

\section{deflection angle}

The reconstructed region is represented by $K=N \times N$ rectangular elements, If there is a total of $q$ projections and in each projection there are $r$ rays, then the total projection number is $m=q \times r$. Equation(5) can transform the partial differential equation about deflected projection into a linear system of optical path difference projection

$$
\sum_{j=1}^{K} A_{i j} f_{j}=p_{i} \quad i=1,2, \ldots, m
$$

where $f_{i}$ represent discrete refraction, $A_{i j}$ is the length of intersection of the $i$ th ray with the $j$ th grid, $p_{i}$ represent the optical path difference.

\subsection{Total Variation (TV) algorithm}

Compressed sensor theory indicates that the original distribution can be reconstructed from a small number of observation values if the distribution itself or in a transformation domain has sparse representation. Here, $\Psi$ represents a sparse transformation, and $A$ represents the corresponding projection matrix. It should be noted that $\Psi$ and $A$ are irrelevant here. The original distribution can be reconstructed by solving equation (7)

$$
\min _{f}\|\Psi f\|_{0}, \text { s.t.Af }=P
$$

In the formula, the zero norm $\|\bullet\|_{0}$ represents the number of non-zero elements . Formula (7) is a typical non-convex optimization problem, which is quite difficult to solve. Therefore, Candes and Donoho proposed to replace $l_{0}$ norm with $l_{1}$ norm ${ }^{[10-11]}$, and equation(7) became equation (8),

$$
\min _{f}\|\Psi f\|_{1}, \text { s.t.Af }=P
$$

In the formula, $\|f\|_{1}=\sum_{i=1}^{n}\left|f_{i}\right|$, then, the above equation (8) can be transformed into a convex optimization problem to solve. On the premise that the sparse matrix and the perceptive matrix are not related, the solutions of equations (7) and (8) are equivalent.

According to CS theory and the principle of deflection angle transformation, combined with the sparseness of physical quantity (temperature, density) distribution, When the sparse transform is a finite difference, it is usually measured by Total Variation, $\|\Psi f\|_{1}$ in equation (8) becomes $\|f\|_{T V}$, as shown in formula (9)

$$
\min _{f}\|f\|_{T V}, \text { s.t. A } f=P
$$

The TV algorithm estimates the total variation of the minimum refractive index by using the gradient descent method, the gradient descent method can be expressed as

$$
f^{(1+1)}=f^{(1)}-\alpha d \frac{v_{T V}}{\left\|v_{T V}\right\|}
$$

Where: $\alpha$ is the relaxation factor of gradient descent method, $d$ is the scale parameter of gradient descent, $l$ is the iterative index, $v_{T V}$ is its gradient descent direction.

\subsection{Diagonal Total Variation(TV) algorithm}

Diagonal TV(DTV) is based on TV. For TV, it uses gradient operators in the $\mathrm{X}$ and $\mathrm{Y}$ directions to sparse represent the measured field, while diagonal TV uses gradient operators in the diagonal direction to sparse represent the measured field. Therefore, the total variation of diagonal TV is defined as follows:

$\|f\|_{D T V}=\sum_{s, t} \sqrt{|f(s-1, t-1)-f(s, t)|^{2}+|f(s-1, t+1)-f(s, t)|^{2}}(11)$

$f$ is the distribution of refractive index, $s, t$ is the ordinal number of the matrix. Similar to TV, minimization of diagonal variation (DTV) can be converted to the following optimization problems:

$$
\min _{f}\|f\|_{D T V}, \text { s.t.A } f=P
$$

The above optimization problem using the gradient descent method can be expressed as:

$$
f^{(k+1)}=f^{(k)}-\beta d \frac{v_{D T V}}{\left\|v_{D T V}\right\|}
$$

Where: $\beta$ is the relaxation factor of gradient descent method, $d$ is the scale parameter of gradient descent, $k$ is the iterative index, $v_{D T V}$ is its gradient descent direction. the implementation steps of DTV algorithm can be obtained:

1)Input the measurement angle projection data and initialize the reconstructed field.

2)Using ART algorithm to update and reconstruct the measured field.

3)According to formula (10), the gradient descent method is used to minimize the total variation (TV) of the reconstructed field

4) When there is no obvious change in the reconstruction iteration of $\mathrm{TV}$, according to formula (11), the gradient descent method is used to minimize the DTV of the reconstructed field. 
5)Repeat steps 2),3)and 4), stop the iteration until the convergence condition of the reconstruction iteration is met.

\section{Computer Numerical Simulations}

\subsection{Simulated Temperature Field}

To investigate the reliability and quality of the DTV algorithm, a three-peak Gaussian temperature field $t(x, y)$, shown in figure 2 , is selected. It covers a square region of $30 \mathrm{~cm} \times 30 \mathrm{~cm}$ and is divided into $30 \times 30$ small cells. Equation (14) is the temperature distribution

$$
\begin{array}{r}
t(x, y)=150 \exp \left[-\frac{(x-10)^{2}+(y-20)^{2}}{10}\right] \\
+200 \exp \left[-\frac{(x-20)^{2}+(y-12)^{2}}{10}\right] \\
+200 \exp \left[-\frac{(x-8)^{2}+(y-8)^{2}}{15}\right]
\end{array}
$$

One sampling geometry is used for the numerical reconstructions: six projection views evenly spaced over a $180^{\circ}$ total view at intervals of $30^{\circ}$. In the field reconstruction based on diagonal $\mathrm{TV}$, the sampling points per view $\mathrm{r}$ are 40, and we chose $\lambda=0.9, \alpha=0.55, \beta=0.28$. One constraint, that is, $\hat{t}_{i} \geq 0$ are incorporated into each iteration. The number of iterations of $\mathrm{TV}$ is $50, \mathrm{TV}+\mathrm{DTV}$ is first replaced by TV method for 30 times, and then by DTV for 20 times. After the refractive index distribution is reconstructed, the temperature distribution is derived from the G-D (gladstone-dale) formula.

Two reconstruction error measures are introduced to evaluate the reconstruction qualities. They are the root mean square error (RMSE), and peak value error (PVE):

$$
\begin{aligned}
& R M S E=\frac{\left[\sum_{i=0}^{N-1}\left(\hat{t}_{i}-t_{i}\right)^{2}\right]^{1 / 2}}{N \bullet t_{\max }} \\
& P V E=\frac{\left|\hat{t}_{\max }-t_{\max }\right|}{t_{\max }}
\end{aligned}
$$

Where $t_{i}$ is the simulated value at the sampled point and $t_{\max }$ is the maximum value; $\hat{t}_{i}$ is the reconstruction value sampled at the same point, and $\hat{t}_{\max }$ is the maximum value.

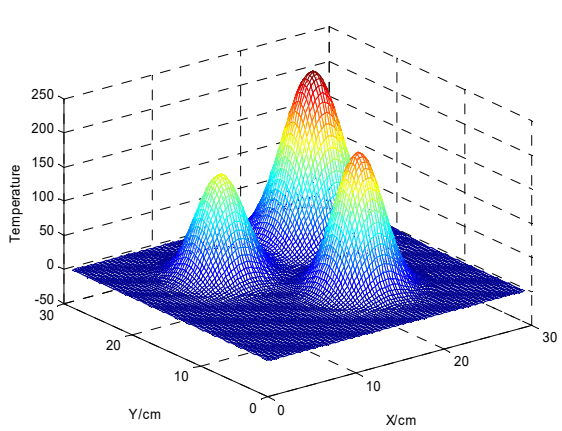

Figure 2. Three-peak Gaussian simulated temperature field.

\subsection{Reconstruction results and analysis}

The ambient refractive index $n_{0}$ is 1.00029 . The best reconstruction results from the TV and TV+DTV algorithms are shown in Figure 3, The RMSE convergence of the TV and TV+DTV algorithms are shown in Figure 4.

Under the 6 projection angles, the comparison between figure 4(a) and figure 4(b) show that there is no significant difference between TV and TV+DTV. In order to further verify the advantages and disadvantages of the algorithm, We performed experiments to obtain RMSE and PVE curves of TV algorithm and TV+DTV algorithm, as shown in figure 4 , It can be seen from the figure that when we replaced the TV constraint with DTV constraint in 30 iterations, RMSE and PVE get a jump change, and smaller RMSE and PVE are obtained, indicating that the reconstruction quality is higher, while the TV algorithm remains stable after 30 iterations without significant changes.

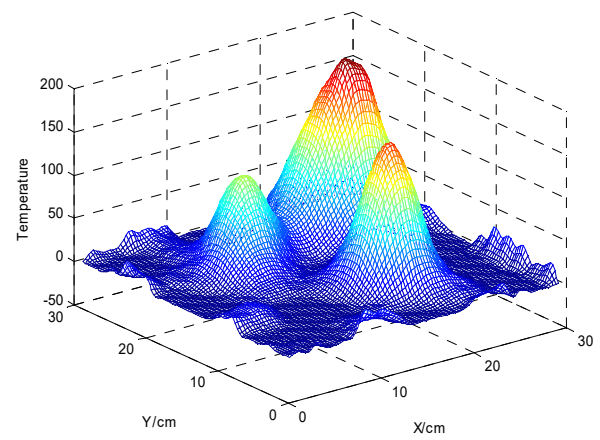

(a)

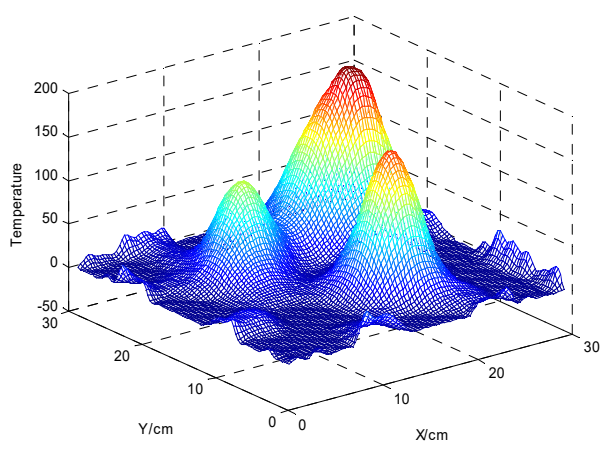

(b)

Figure 3. Reconstructions of the three-peak Gaussian temperature fields: (a) TV, (b)TV+DTV 


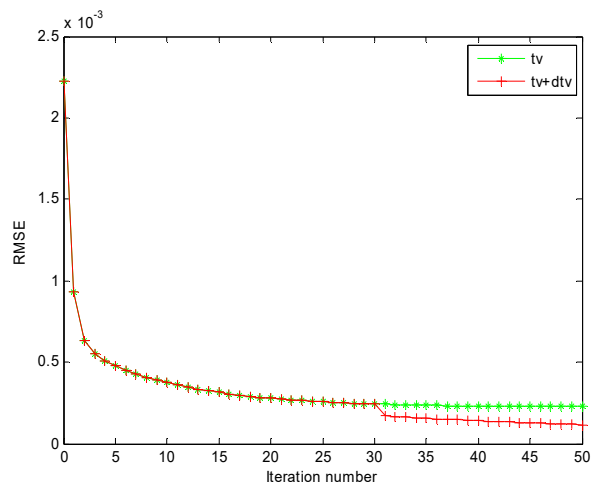

(a)

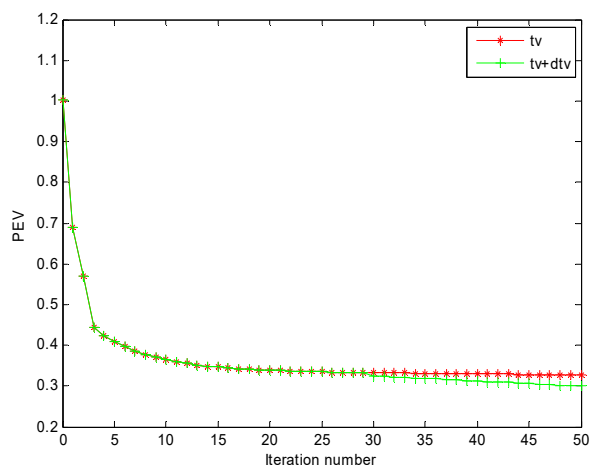

(b)

Figure 4.Comparison of reconstruction errors resulting from different algorithms: (a) root mean square error (b) peak value error

\section{Conclusions}

CS is a theory and method that the original field can be reconstructed with spare projection. In this paper, it is applied to deflection tomography reconstruction algorithm. Experimental results show that the TV+DTV algorithm is not different from the TV method in terms of overall visual effect, however, in the case of limited projection data, the TV+DTV algorithm is significantly better than the TV algorithm in RMSE and PEV, indicating that the TV+DTV algorithm is more suitable for reconstruction in the case of sparse sampling.

\section{References}

1. Lewitt. R. M., Reconstruction algorithms: transform methods, Proc.IEEE 71, 390 408 (1983).

2. Y.Censor, Finite series expansion reconstruction methods, Proc. IEEE 71, 409 419 (1983).

3. J. Stricker, E. Keren, O. Kafri. Axisymmetric density field measurements by Moire deflectometry.AIAA,21,1767 1769 (1983)

4. J. Stricker. Analysis of 3-D phase objects by Moire deflectometry, Applied Optics, 23, 3657 3659 (1984)

5. M Wang ; L Ma ; D Li ; J Zhong, Subfringe integration method for automatic analysis of Moiré deflection tomography. Optical Engineering, 39, 2726 2733(2000)
6. Gregory W. Faris, Robert L. Byer. Three-dimensional beam-deflection optical tomography of a supersonic jet. Applied Optics, 27, 5202 5212(1988)

7. Dapeng Yan, Anzhi He, Xiaowu Ni. New method of asymmetric flow field measurement in hypersonic shock tunnel. Applied Optics, 30,770 774(1991)

8. O.Kafri, Moiré deflectometry: a ray deflection approach to optical testing, Opt. Eng. 24, 944 960 (1985)

9. D.Zhu,Laser Metrology for Thermal Physics (Science,1990)

10. Candés E J,Wakin M. An introduction to compressive sampling. IEEE Sign Processing Magazine.25,1 30(2008).

11. Donoho D. Compressed sensing. IEEE Transactions on information Theory. 52,1289 -1306(2006). 